\title{
ROSAT WFC EUV OBSERVATIONS OF AB DOR (AND OTHER LOCAL GROUP STARS)
}

\author{
BARRY J. KELLETT \& GORDON E. BROMAGE \\ Rutherford Appleton Laboratory, United Kingdom
}

\section{INTRODUCTION}

The Rosat Observatory was launched on June $1^{s t}, 1990$, and after a brief Performance Verification phase, performed an almost complete sky survey. One of the two main telescopes on the Observatory is the UK Wide Field Camera (WFC). The WFC is the first extreme ultraviolet (EUV) instrument to survey the whole sky, and during the survey detected several hundred active late-type stars.

The WFC (Wells et al., 1990) was built by a consortium of five UK institutes, four Universities and the Rutherford Appleton Laboratory. During the allsky survey, two filters were used. These allow broad-band photometry to be performed. The S1A filter (lexan/carbon/boron) and S2A filter (lexan/beryllium) were alternated on a one day basis. The S1A filter covers the energy range $0.085-0.180 \mathrm{KeV}$, centred on $0.124 \mathrm{KeV}$, while the softer S2A filter covers the range $0.070-0.110 \mathrm{KeV}$ (centred on $0.090 \mathrm{KeV})(10 \%$ efficiency) [see Kent et al. (1990) for a full description of the WFC filters].

Because of the way that the survey was performed any given EUV source would be observed for a minimum of 5 days. However, the data would be composed of brief "snapshots" of the source lasting 30-80 seconds, separated by one Rosat orbit (about 96 minutes). Therefore, the typical exposure time is about 1000 seconds in each of the two survey filters.

\section{AB Doradus (HD36705)}

AB Dor is a rapidly rotating young star which reveals its magnetic-related activity in a variety of ways. In particular, it is a strong X-ray source, with flares observed by the Einstein HRI, EXOSAT ME, and Ginga LAC [Vilhu \& Linsky (1987), Collier-Cameron et al. (1988), Vilhu et al. (1991)]. Ginga was also able to show some marginal evidence for X-ray modulation with the 0.514 day rotation period of $\mathrm{AB}$ Dor.

In the Rosat All-Sky survey, AB Dor is nearly "circumpolar" and was visible for about 2 months (in the WFC) of the 6 month survey. During much of the survey, AB Dor would have been viewed from about the same point in the Rosat orbit. Unfortunately, this point is quite close to the southern auroral particle zones of the Earth, and consequently, the WFC detector was switched off for much of the potential survey time of AB Dor, and with quite a high background rate during much of the remaining time. This leads to a good deal of scatter in the data (although some of the scatter must also be due to the activity of AB Dor itself). However, AB Dor was detected as a very significant source in both the survey filters. The question of whether the survey data shows any modulation 
on the 0.514 day rotation period of $A B$ Dor is more difficult to establish because of the problems mentioned above. But it is possible that some modulation was present for at least part of the time. This conclusion was also reached by Kürster et al. 1992, from the Rosat XRT survey data.

PZ Tel (HD174429)

Another well known fast rotator is PZ Tel. Unfortunately, the WFC survey of $\mathrm{PZ}$ Tel was badly affected by data loss (similar to AB Dor), and only received a relatively short exposure (see Table 2). It is also much further away than $\mathrm{AB}$ Dor $(68 \mathrm{pc}$ versus $20 \mathrm{pc})$. Consequently, no EUV flux was detected from PZ Tel

"Speedy Mic" - the new fast rotator

During the WFC survey, many previously unknown late-type stars were found to be EUV emitters. Optical follow-up spectroscopy has confirmed many of them to be very active indeed. One of the most interesting of the early discoveries was a new source in the southern constellation Microscopium. The new source was quickly christened "Speedy" for reasons that will become apparent. The source was clearly variable during the survey. In fact, detailed analysis has shown that the S1 and S2 detections of this source are due entirely to the presence of a long-lasting flare on the star, with $N O$ quiescent emission being detected from Speedy (Bromage et al., 1992).

The WFC position lies very close to a catalogued $9^{\text {th }}$ mag K0 star (HD197890). Optical follow-up spectra of Speedy taken with the $1.9 \mathrm{~m}$ SAAO telescope with the RPCS detector clearly indicated broad emission lines of $\mathrm{Ca} \mathrm{II} \mathrm{H}+\mathrm{K}$ in the spectrum of Speedy. We estimate a (deconvolved) FWHM of $\sim 230 \mathrm{~km} / \mathrm{s}$, which is faster than AB Dor.

Further optical monitoring of Speedy has failed to show any signs of binarity, so we have concluded that this is a very rapidly rotating single $K$ dwarf. It also shares the same common proper motion shown by AB Dor and PZ Tel. In fact all $3 \mathrm{~K} \mathrm{dwarfs}$ are members of the Pleiades or Local group (see Innis et al., 1986, Bromage et al., 1992).

\section{The Local Group K dwarfs}

In recent years, 4 other $K$ dwarfs have been associated with the Local Group (see Anders et al., 1991). These stars are listed in Table 1, along with the stars already discussed. Although these $4 \mathrm{~K}$ dwarfs all show much smaller vsin $i$ 's than the stars already discussed above (based on their relative photometric periods, given in Table 1, for example), all 4 were clearly detected in the survey. Table 2 summaries the WFC EUV observations of these $\sim \mathrm{KOV}$ stars. In all 4 cases, detailed analysis of the data has shown that these detections are "quiescent" emission from these stars; ie. there are no significant variations present in the data which might indicate the presence of flares. 
TABLE 1: Local Association K Stars

\begin{tabular}{|l|c|c|c|c|c|}
\hline HD Number (Name) & $\mathrm{V}$ & Sp.Typ. & $\mathrm{P}_{p h o t}(\mathrm{~d})$ & $\mathrm{P}_{\text {orb }}(\mathrm{d})$ & $\mathrm{d}_{p c}$ \\
\hline 17433 (VY Ari) & 6.87 & G9Ve & 17.4 & 13.20 & 21 \\
36705 (AB Dor) & 6.95 & K1-2III-IVp & 0.514 & - & 20 \\
82558 & 7.50 & $\mathrm{dK} 0$ & 1.66 & - & 13 \\
102077 (V838 Cen) & 8.97 & $\mathrm{~K} 0-1 \mathrm{Vp}$ & 1.84 & - & 42 \\
139084 (V343 Nor) & 8.14 & $\mathrm{~K} 0 \mathrm{~V}$ & 4.2 & - & 55 \\
174429 (PZ Tel) & 8.45 & $\mathrm{~K} 0 \mathrm{III}-\mathrm{VP}$ & 0.945 & - & 68 \\
197890 ("Speedy") & 9.3 & $\mathrm{~K} 0 \mathrm{~V}$ & 0.338 & - & $\sim 40$ \\
\hline
\end{tabular}

TABLE 2: WFC Survey Results

\begin{tabular}{|l|c|c|c|c|}
\hline \multirow{2}{*}{ Star } & \multicolumn{2}{|c|}{ S1A Filter } & \multicolumn{2}{c|}{ S2A Filter } \\
\cline { 2 - 5 } & Survey (s) & Cts/Sec & Survey (s) & Cts/Sec \\
\hline VY Ari & 1261.2 & 0.094 & 849.0 & 0.096 \\
AB Dor & 13442. & 0.075 & 12539. & 0.090 \\
HD82558 & 1955.2 & 0.033 & 2106.7 & 0.028 \\
V838 Cen & 954.2 & 0.025 & 861.8 & 0.030 \\
V343 Nor & 1261.5 & 0.021 & 1780.6 & 0.040 \\
PZ Tel & 589.5 & $<0.009$ & 710.3 & $<0.034$ \\
Speedy & 1126.0 & 0.030 & 1302.7 & 0.072 \\
\hline
\end{tabular}




\section{CONCLUSION}

The WFC EUV survey results presented here show that the Local Association $\mathrm{K}$ dwarfs are strong EUV emitters. The stars in the association are thought to be 50-70 million years old, and it would seem it is these stars relatively young age that make them so active; since the "slow" rotators, like V343 Nor and VY Ari, appear just as strong as the rapid stars, like AB Dor and Speedy. In fact, as already mentioned, Speedy Mic was NOT seen in quiescence, whereas the 4 $\mathrm{K}$ dwarfs added to this study were $A L L$ clearly detected in quiescence.

\section{ACKNOWLEDGMENTS}

The Wide Field Camera is supported by the UK Science and Engineering Research Council. The WFC instrument and associated Ground Systems were developed by a consortium of five UK institutes: the University of Leicester, SERC's Rutherford Appleton Laboratory, Imperial College STM London, the University of Birmingham, and the Mullard Space Science Laboratory of University College London. The authors would like to thank all WFC personnel at the Consortium Institutes involved in the design and development of the Instrument. This work utilised STARLINK software and facilities at the Rutherford Appleton STARLINK node.

\section{REFERENCES}

Anders, G.J., Innis, J.L., Coates, D.W., \& Thompson, K., 1991, MNRAS, 252, 408.

Bromage, G.E., et al., 1992, PASP Conf. Ser. (Proc. Tucson Workshop, Oct. 1991), in press.

Collier-Cameron, A., et al., 1988, MNRAS, 231, 131.

Innis, J.L., Thompson, K., \& Coates, D.W., 1986, MNRAS, 223, 183.

Kent, B.J., et al. 1990, Proc. S.P.I.E., 1344, 255.

Kürster, M., et al., 1992, PASP Conf. Ser. (Proc. Tucson Workshop, Oct. 1991), in press.

Vilhu, O., \& Linsky, J.L., 1987, PASP, 99, 1071.

Vilhu, O., Tsuru, T., \& Collier-Cameron, A., 1991, "Iron Line Diagnostics in $X$-ray Sources”, Eds. Treves, A., Perola, G.C., \& Stella, L., 30.

Wells, A., et al. 1990, Proc. S.P.I.E., 1344, 230. 